\title{
Panorama de la ArQueología Amazónica DEL ECUADOR. PRIMERA APROXIMACIÓN
}

\author{
A. Jorge Arellano López'
}

E ste trabajo presenta el actual conocimiento del desarrollo cultural prehispánico en el Alto y Bajo Amazonas del Ecuador. El incremento de la exploración petrolera durante las dos últimas décadas, también fue favorable para el incremento de reconocimientos arqueológicos, en especial en la Amazonía Baja. El nuevo panorama arqueológico presentado en este trabajo tiene base, primero en la descripción de los restos de cerámica y segundo en el patrón de asentamiento arqueológico en la Alta y Baja Amazonía.

This paper examines the current knowledge of the prehispanic cultural development in the high and lower Ecuadorian Amazon. The increase of oil exploration during the last two decades also was favorable to an increase ofarchaeological surveys, especially on the lower Amazon. The new archaeological panorama presented in this paper has its base first, in the description of remains of pottery and secondly on the archaeological settlement pattern in the high and lower Ecuadorian Amazon.

Trabajo Presentado al Primer Simposio de Arqueología Amazónica del Perú, organizado en Marzo del 2006 por la Escuela de Arqueología de la Universidad Nacional Mayor de San Marcos, el Centro Cultural José Pío Aza y Centro Cultural de la Casa de San Marcos. Lima, Perú. 
En las últimas décadas los estudios medioambientales realizados en la Amazonía ecuatoriana, favorecieron el desarrollo de una arqueología amazónica con un alto grado de sofisticación. Estos estudios que tienen como finalidad ofrecer una alternativa para evitar un excesivo deterioro y proporcionar una aceptable conservación del medioambiente, también dieron lugar a la obtención de un apreciable porcentaje de datos arqueológicos que paulatinamente van cambiando el panorama del conocimiento cultural prehispánico.

La Amazonía ecuatoriana, desde el ingreso de Gonzalo Pizarro y Francisco de Orellana en 1541, ha despertado una serie de mitos relacionados con la existencia de grandes poblaciones nativas a lo largo del curso, primero del Río Napo y más adelante del Río Amazonas. En la actualidad el principal objetivo de la arqueología ecuatoriana amazónica tiene relación con la configuración de un panorama cronológico del desarrollo cultural, y al mismo tiempo determinar el periodo en el cual se iniciaron los contactos que pudieron tener las antiguas sociedades amazónicas con las asentadas en la sierra.

La periodificación cronológica del desarrollo cultural de las sociedades nativas prehispánicas en el Ecuador se inicia con el Arcaico, del cual muy pocas evidencias se encuentran en las tierras amazónicas. El Formativo, adolece también de limitaciones y está mayormente referido a la Fase Cosanga en la ceja de montaña oriental y Fase Pastaza en la Amazonía baja del Sur. Los periodos siguientes, denominados Desarrollos Regionales e Integración, tienen mayor trascendencia en la Amazonía baja del Norte por la presencia de fases culturales que actualmente se encuentran en una secuencia bien definida.

En este sentido, el propósito de la presente síntesis es presentar una primera aproximación al panorama del desarrollo cultural prehispánico en la Amazonía de Ecuador, tomando como base las investigaciones arqueológicas efectuadas en esta importante región. Esperamos que sea un aporte inicial a la interpretación y reconstrucción económica social de las diferentes culturas y su interacción con el medioambiente.

\section{Cuadro geomorfológico e historia arqueológica}

La región amazónica de Ecuador está usualmente dividida en dos subregiones: la primera, denominada Alta Amazonía (comprende entre los 1000 y 600 $\mathrm{msnm}$ ), es una franja paralela que corre de Norte a Sur al pie de la ceja de montaña de las estribaciones orientales de la cordillera de los Andes. Esta franja está constituida por un paisaje de valles asociados a serranías bajas y colinas, cubiertas con una vegetación típica de bosques tropicales húmedos. Los ríos forman parte del drenaje de la gran cuenca del Río Amazonas. 
Las principales cuencas son por el Norte los ríos Aguarico y Napo que conforman un amplio abanico de afluentes con dirección al Este, y por el Sur las cuencas de los ríos Pastaza, Morona y Santiago que se dirigen hacia el Río Marañón formando a su vez otra extensa ramificación de afluentes al Sureste. En la segunda sub-región, Baja Amazonía (600 a 200 msnm), la topografía del paisaje tiene como característica una ondulación del terreno, en la cual resaltan las colinas que se ubican en los alrededores de la ciudad de Lago Agrio y la presencia de terrazas altas y bajas a lo largo de los canales de los ríos Aguarico, Napo y Pastaza. Las zonas inter-fluviales entre el Aguarico y el Napo, y el Napo y Pastaza, está conformada por planicies aluviales generalmente sujetas a inundaciones periódicas, las cuales posteriormente forman áreas cubiertas por pantanos en los que sobresalen los altos topográficos del terreno ondulado. Esta particularidad es más apreciable al Sur del Río Napo donde los tributarios divagan en la planicie formando meandros que constantemente son abandonados y pasan a formar lagunas semi-circulares.

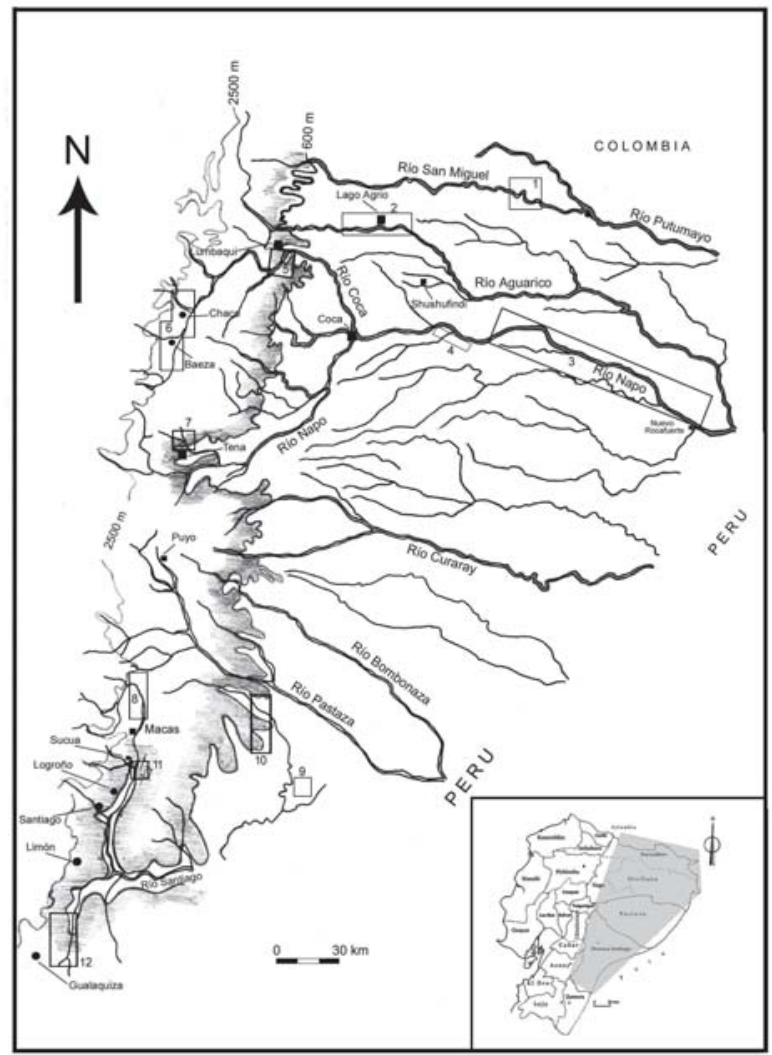

Figura 1.

Ubicación regional de las áreas de investigación arqueológica en Ceja de Montaña, Alto y Bajo Amazonas del Ecuador. 
1) Aguilera 2002, 2) Arellano 1999, 2002; Carrera 2002, 3) Evans y Meggers 1968, 4) Netherly 1997, 5) Salazar et. Al. 1999, 6) Porras 1975; Salazar et al. 1999, 7) Porras 1975, 8) Porras 1987, 1989; Salazar 1999; Rostain 1999, 9) Rostoker 1966), 10) Porras 1985, 11) Porras 1985, 12) Lerdergerber 2006.

La historia arqueológica de la Amazonía del Ecuador se inicia con las investigaciones realizadas por Clifford Evans y Betty J. Meggers (1968) a lo largo del Río Napo. Los sitios arqueológicos ubicados durante su trabajo de campo proporcionaron las primeras evidencias de la existencia de culturas prehispánicas al margen de las descritas en las crónicas y reportes de exploradores y viajeros. Evans y Meggers (1968), mediante el análisis de los datos de campo, y la clasificación tipológica del material cerámico que permitió la elaboración de secuencias seriadas en asociación a dataciones radiocarbónicas, pudieron determinar la presencia sucesiva de cuatro Fases Culturales en la Baja Amazonía. La más temprana Yasuni (0 AD), Tivacundo (510 d.C.), Napo (1168-1480 d.C.) y por último Cotacocha, que prácticamente corresponde a la etapa de la conquista española.

Las dos primeras fases, Yasuni y Tivacuno, se encuentran ubicadas en la cronología cultural del Ecuador en el período de Desarrollos Regionales y la Fase Napo en el periodo de Integración. Los rasgos diagnósticos de la cerámica de la Fase Yasuni tienen relación con el uso de dos variedades de temperante o desgrasante, la una es mezcla de cariapé con partículas de carbón y la otra arena. Las formas están asociadas a cuencos, platos de perfiles simples, y vasijas abiertas con perfiles compuestos. La decoración más frecuente son incisiones formando zonas en el cuerpo de las vasijas. La Fase Tivacuno está caracterizada por cuencos y vasijas semiesferoidales, decorada con diseños geométricos definidos por incisiones sobre un engobe rojo.

Por su parte, la Fase Napo es la más representativa de las culturas amazónicas ecuatorianas por la variedad de formas de sus vasijas con decoración polícroma (blanco, rojo y negro), entre las que se destacan urnas funerarias y vasijas con decoración excisa o acanalada. Este material cerámico está complementado con la presencia de sellos y hachas líticas. Finalmente, la Fase Cotacocha ubicada en un solo sitio cercano a la frontera con Perú, tiene formas consideradas utilitarias: cuencos con base anular y vasijas globulares abiertas o con cuello y bordes expandidos. La decoración está restringida a bandas rojas limitadas por líneas incisas (Evans y Meggers, 1968: 87). Los sitios estudiados por Evans y Meggers se ubican entre las localidades de Edén y Nuevo Rocafuerte, y con excepción de dos sitios que se encuentran en la orilla izquierda del Napo, los demás se sitúan en la orilla derecha. 


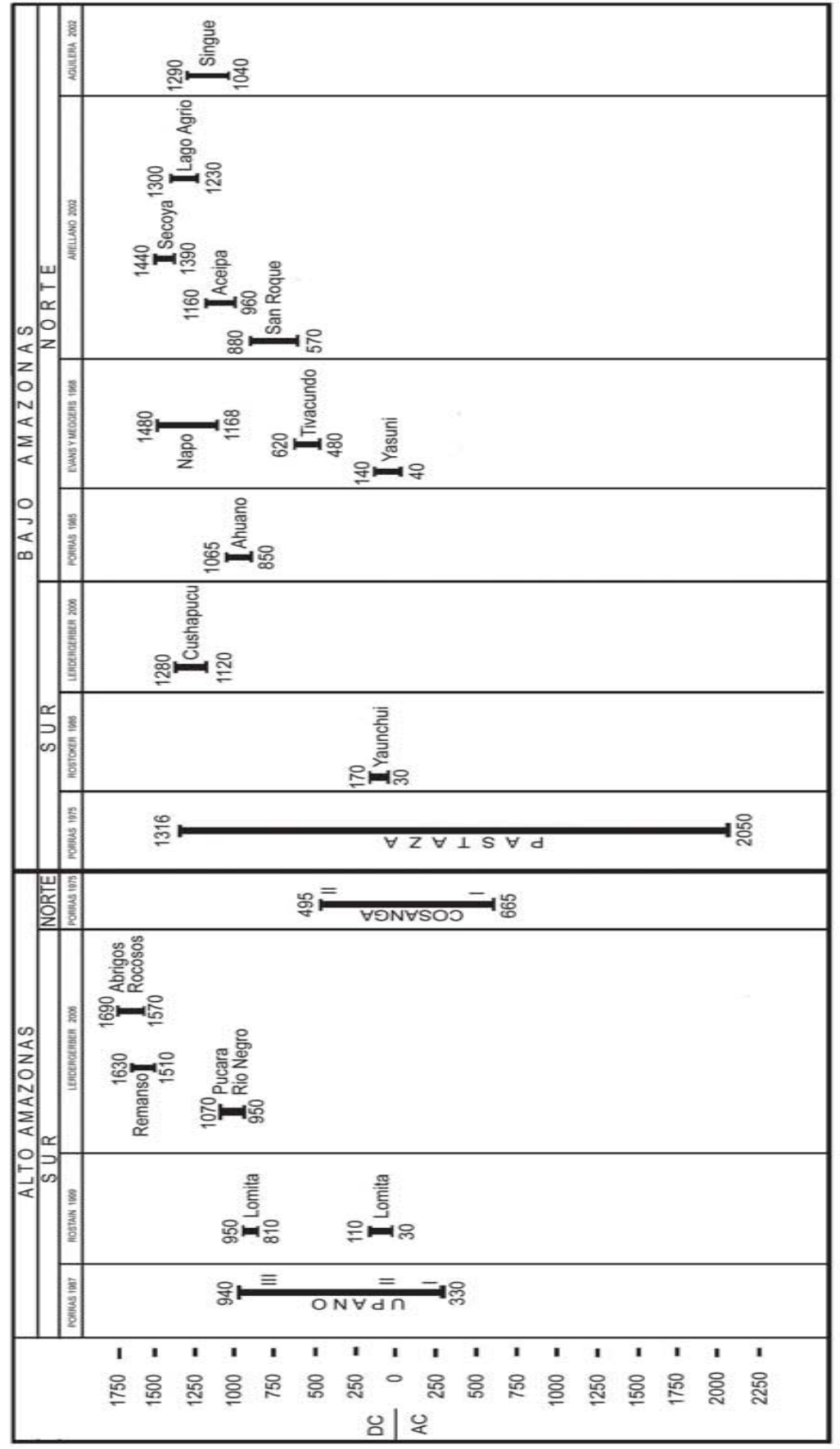

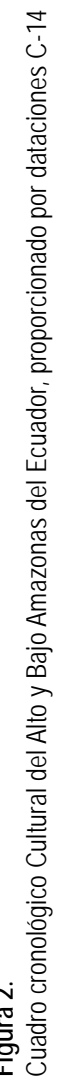


A partir de esta secuencia, las investigaciones del Padre Porras $(1975,1975 b$, 1978, 1985 y 1987) se concentraron particularmente en la Ceja de Montaña y en la Alta Amazonía. Las áreas geográficas que proporcionaron nuevos elementos de juicio para la interpretación de la interacción social de las culturas de la ceja de montaña con los pueblos prehispánicos amazónicos, fueron las que giran alrededor de las poblaciones de Baeza, Tena y Macas. En cada una de ellas, Porras determinó que las culturas tenían una mayor organización social que dio lugar a la configuración de centros con arquitectura doméstica y ocasionalmente monumental.

En la ceja de montaña Norte, la Fase Cosanga (600 a.C. a 700 d.C.) del periodo de Desarrollos Regionales, está caracterizada por una cerámica de paredes delgadas con temperante o desgrasante de arena fina micácea. Entre las formas de las vasijas se destacan ollas globulares con cuello y bordes evertidos, cuencos con pedestal y figuras antropomorfas de cerámica como parte de urnas funerarias. Esta fase abarca un territorio que comprende las actuales poblaciones que se ubican en el valle del Río Quijos.

Al Sur la Fase Upano, cuyo principal sitio se encuentra situado en la zona de Huapula, cercana a la población de Macas (provincia Morona Santiago), es importante por la calidad de sus restos cerámicos y por la configuración arquitectónica estructural que presenta su asentamiento. Porras (1987: 38-40) menciona que el sitio está compuesto por 180 plataformas o pirámides truncadas, subdividido en 26 complejos. Las principales formas de las vasijas Upano son platos carenados de paredes evertidas con dos variedades de bases: cóncavas con botones y planas o convexas con pedestal. En menor porcentaje se encuentran ollas carenadas, ollas con cuello y cuencos. La decoración es geométrica de color rojo en bandas limitadas por incisiones. También existen fragmentos con decoración incisa, corrugada y otros con decoración en negativo. En el contexto arqueológico de Upano es frecuente la presencia de figurinas de cerámica, sellos circulares, y restos de botellas con asa puente. Mediante la clasificación tipológica de la cerámica, la conformación de frecuencias seriadas y la inclusión de dataciones radiocarbónicas. La Fase Upano fue subdividida en tres periodos: Upano I (1100 a.C. al 120 d.C.), Upano II (40 d.C. al 170 d.C.) y Upano III (640 d.C. al 940 d.C.). Al margen de los trabajos en el complejo central, Porras (1987: 72) menciona que realizó excavaciones de sondeo en nueve sitios de los cuales los más importantes fueron Chiguaza y San Paulo.

De igual manera, en el bajo Amazonas de la zona Sur, Porras (1975) encontró evidencias de una fase formativa en las orillas del Río Huasaga a la que denominó Fase Pastaza (2000 a.C. al 1000 a.C.). Esta fase está compuesta por una cerámica decorada con técnicas bien depuradas entre las que se destacan el inciso y punteado zonal. En el complejo Pastaza también se puede observar fragmentos con decoración corrugada, ungulada y con engobe rojo. Sin embargo, las formas son muy comunes, cuencos, vasos de perfiles simples y algunas vasijas de cuerpo esferoidal con cuello. 
Por otra parte, los trabajos de Athens (1984) en el sitio Pumpuentza 1, en la cuenca del Río Macuma, determinaron que los depósitos culturales se encuentran usualmente hasta una profundidad de $20 \mathrm{~cm}$. El material cultural cerámico recuperado en este sitio presenta un apreciable porcentaje de fragmentos con una decoración incisa zonal con diseños variados y tendrían similitud con los tipos Pastaza Inciso y Pastaza Puntuado de la Fase Pastaza (Athens, 1984: 131). Sin embargo, la discrepancia con la Fase Pastaza está referida a su ubicación cronológica, puesto que el sitio Pumpuentza 1 tiene una fecha radiocarbónica de $740 \pm 65$ d.C. y no se trataría de una fase formativa.

De manera complementaria para esta región de Morona Santiago, se puede mencionar que en el transcurso de las investigaciones etnográficas de la etnia Shuar (Jívaro) realizadas en el año 1953, M. Harner ubicó el sitio arqueológico Yaunchu en el cantón Sucua. El sitio Yaunchu se encuentra en la orilla derecha del Río Tutanazoga, que es tributario del Río Upano. El material cultural cerámico y lítico obtenido por Harner (1973) mediante la excavación de una cuadrícula de más de dos metros de profundidad, fue re-analizado por A. Rostoker en 1988 y luego sus resultados parcialmente publicados en 1996. En el material cerámico se puede observar que los tipos importantes son el decorado Inciso en Bandas Rojas, Puntuado e Inciso. Las formas comunes son platos carinados, ollas con cuello y cuencos. Según Rostoker (1996: 22) la ubicación cronológica de este complejo presenta problemas por la procedencia de las muestras de carbón. La fecha más aceptada es 70 +/- 100 AD y probablemente proviene de la parte inferior de la primera capa. La presencia de la cerámica tipo Inciso en Bandas Rojas permitió la correlación con el sitio de Pirincay situado en el valle del Río Paute en la sierra del Azuay (Brunhs, 1994: 142), y que desemboca en el Río Upano. Este tipo de cerámica estaría presente por vez primera entre el 200 a.C. y 50 AD confirmando eventualmente que el sitio Yaunchu fue ocupado alrededor del 70 +/- 100 AD (Rostoker, 1996: 23).

Finalmente, Porras (1978) menciona evidencias de material cultural cerámico y de concha en la cueva de Los Tayos, situado al suroeste de la ciudad de Macas en la provincia Morona Santiago, cercano a los límites internacionales con la República del Perú. Los tipos de cerámica identificados fueron: rojo pulido, rojo sobre leonado, negro sobre leonado, inciso y punteado. La ubicación cronológica de este material «de ofrenda» fue determinada en base a «datación radiocarbónica en conchas y de termoluminiscencia en cerámica», los mismos que indican que el sitio fue utilizado a los 1500 a. C. y que corresponde al Periodo Formativo (Porras, 1978; 1987: 228).

\section{Las nuevas evidencias en la Amazonía}

A partir de la mencionada secuencia cultural, las posteriores investigaciones trataron de integrar los nuevos sitios y los hallazgos aislados de evidencias arqueológicas a la cronología y fases establecidas en la Amazonía Alta y Baja, en particular a la secuencia del Río Napo. 
En la franja Norte de la Alta Amazonía no se tienen trabajos de investigación que hayan sido publicados. Sin embargo, existen una serie de informes de reconocimientos y excavaciones arqueológicas de varios sitios que estuvieron involucrados en el desarrollo de líneas petroleras. Estos informes indican que entre Baeza y El Tena se encuentran varios asentamientos rodeados por murallas defensivas y al parecer los restos culturales corresponden a la Fase Cosanga. Lamentablemente los resultados de estos trabajos no son de dominio público por las restricciones que tienen las compañías petroleras que operan en la zona.

Uno de los informes más importantes se refiere a las investigaciones arqueológicas efectuadas en las diferentes transectas propuestas para línea del oleoducto de crudos pesados que conecta la Amazonía Baja y Alta con la sierra y la costa (Salazar et al, 1999). En el sector que comprende la Amazonía Alta se pudo obtener los mejores datos que se tiene al presente sobre la presencia de restos arqueológicos. Estos datos se complementan a los obtenidos por el Padre Porras y permiten formar un panorama cercanamente completo de esta parte.

Entre los pueblos de Gonzalo Pizarro (600 msnm) y Baeza (1800 msnm) se identificaron 26 sitios que corresponden a dos horizontes culturales: el primero se encuentra conformado por una cerámica con decoraciones excisas, incisas, corrugadas, punteadas y con muescas y el segundo por una cerámica de paredes delgadas y pasta fina con desgrasante micáceo (Salazar et al, 1999: 28-29). El primer horizonte se encuentra en el contacto entre la Alta Amazonía con la Baja Amazonía y estaría tentativamente asociado a la Fase Pastaza, mientras el segundo horizonte está directamente relacionado con la Alta Amazonía y pertenece a la Fase Cosanga.

Entre los complejos culturales de estos sitios es frecuente el hallazgo de material lítico, en el que sobresalen una variedad de hachas y lascas de obsidiana. Este último material es muy importante por su probable origen en la serranía de Quiscatola, lo cual permitiría considerar una variedad de hipótesis con relación a la interacción cultural de pueblos amazónicos con la sierra.

La Baja Amazonía de la zona Norte tiene un mayor porcentaje de investigaciones arqueológicas, pero en general corresponden a escuetos informes sobre la presencia de restos arqueológicos en áreas específicas donde se realizaron trabajos de infraestructura petrolera. Entre estos trabajos se distinguen los estudios efectuados por Netherly (1997), Aguilera (2002), Arellano (2002) y Carrera (2002). El primero se refiere a los patrones de ocupación al Sur del Río Napo y los posteriores a las investigaciones y excavaciones arqueológicas realizadas al Norte del Río Aguarico. Nuevas fases culturales fueron identificadas en las orillas del Napo y zona interfluvial del Aguarico y Napo. Estas fases son: San Roque, Aceipa, Palmeras y Secoya, las tres primeras pertenecen a la tradición polícroma mientras la última corresponde a la tradición corrugada (Arellano, 2007; en prensa). 
En la pequeña sub-cuenca del Río San Miguel que desemboca en el Río Putumayo, se ubicaron una serie de sitios con material cerámico y sitios líticos que son considerados talleres por la abundancia de restos de talla. Estos últimos fueron fechados cronológicamente en el 1100 a. C. al 850 a. C. (Aguilera, 2002: 116). El sitio Singue 1 es el representativo de los que contienen restos cerámicos, los tipos corrugado y falso corrugado están representados en cuencos, ollas carenadas y ollas de bordes evertidos. La datación para Singue 1 tiene un rango entre el 1040 A.D. al 1290 A.D. (Aguilera, 2002: 117). Al Sur de San Miguel, en la orilla izquierda del Río Aguarico las colinas cercanas a la ciudad de Lago Agrio contienen ocupaciones fechadas entre el 1230 A.D. al 1300 A.D., con restos de una cerámica y vasijas con borde reforzado de los tipos engobado en rojo y pulido en negro (Arellano, 2002: 159). En la misma zona, las terrazas bajas presentan sitios cerámicos con los tipos corrugado y falso corrugado asociados a un tipo de cerámica decorada con líneas, bandas y puntos de color rojo sobre un fondo natural (Arellano, 2002: 150; Carrera, 2002: 180). Los tipos corrugado y falso corrugado entrarían en el rango cronológico establecido para Singue 1, mientras el tipo decorado en rojo podría ser de una época más tardía.

Entre otros trabajos realizados en la Amazonía Norte, se pueden mencionar los llevados a cabo en el sitio Bicundo Chico por Bolaños et al. (1999), en los sitios Curiurcu y El Avispal por Delgado (1999) y el sitio Pata 1 por Echeverría (1999). El material cultural de ninguno de estos sitios fue identificado en una fase cultural, pero se tienen dataciones que los sitúan en el periodo Formativo (Rostoker, 2003: 542-43).

Finalmente, los actuales trabajos arqueológicos en la orilla derecha del Napo indican la presencia de otras fases culturales que se sitúan en un rango intermedio entre lo que se denomina Fase Tivacundo y la Fase Napo. Los sitios tienen en general una apreciable extensión superficial y algunos tienen complejos cerámicos que al parecer podrían estar relacionados con actividades ceremoniales.

Al Sur de la Alta Amazonía, un proyecto de re-evaluación de Huapula, sitio tipo de la Fase Upano fue emprendido a partir de 1995 por Salazar (1999), al que estuvieron integradas las excavaciones realizadas por Rostain (1999).

En el sitio Huapula, que tiene «aproximadamente $2400 \mathrm{~m}$ de largo y $300 \mathrm{~m}$ de ancho», Salazar (1999: 183) realizó un estudio regional, encontrando que el complejo principal está asociado a varios sitios por medio de caminos y terraplenes. La configuración planimétrica de cada uno de los sitios fue obtenida por medio de un metódico levantamiento topográfico. De esta forma, se pudo conocer que las estructuras (plataformas) están usualmente dispuestas alrededor de una plaza central y tienen vías de comunicación entre ellas y con el complejo central de Huapula. Por su parte Rostain (1999: 227) realizó excavaciones en el sector denominado La Lomita, 
parte del complejo de Upano - Huapula. Sus resultados complementaron las primeras apreciaciones de Porras $(1987,1989)$ con relación a la presencia de varios periodos de ocupación. El material cerámico obtenido en estas excavaciones tiene directa asociación con el complejo cerámico descrito por Porras (1987). En cuanto a su ubicación cronológica, Salazar (1999: 215), menciona que las dataciones del «depósito de La Lomita» se encuentran en un rango del $40 \pm 70$ d.C. al $880 \pm 60$ d.C. y tienen coincidencia con las fases Upano II y Upano III. Por otra parte, Rostain (1999b) complementó los datos de la Lomita con los obtenidos en la «tola central» del complejo IX situado en el sector Este del sitio Upano - Huapula. Las excavaciones determinaron la presencia de dos niveles culturales separados por una capa de ceniza volcánica. El material cerámico de la capa inferior pertenece a la «cultura Upano» y de manera similar al material de la Lomita, tiene correspondencia con las fases Upano I y II. Según las dataciones es una ocupación entre el 700 a.C. al 400 d.C (Rostain, 1999b.). La capa cultural superior por encima de la ceniza tiene material cerámico y lítico de lo que se vino a llamar «cultura Huapula», fechada entre el 700 d.C. y 1200 d.C. la cerámica representativa de esta cultura es gruesa, decorada con «impresiones digitales- este decorado es llamado corrugado» (Rostain, 1999b: 76-78) y otro tipo decorado en blanco sobre rojo. Las formas de las vasijas son cántaros globulares con cuello y cuencos semiesféricos.

Al Sur de Upano, las investigaciones de Ledergerber-Crespo $(1992,1995,2006)$ estuvieron concentradas en la región de los valles de los ríos Zamora y Santiago, provincia Morona Santiago. Estos trabajos hacen referencia a sitios arqueológicos en el cantón Gualaquiza y se encuentran tanto en la Amazonía Alta como en la Baja. La importancia de estas investigaciones radica en las evidencias del ingreso de las culturas de la sierra hacia la Amazonía. Los sitios: El Remanso, Pucara Río Negro, Finca Rosita, El Empalme, San Juan Bosco y La Selva, que se ubican entre los 1600 y $730 \mathrm{msnm}$ se caracterizan por la presencia de restos arquitectónicos y fragmentos de cerámica. La diferencia entre las sociedades que ocuparon esta particular zona del piedemonte amazónico se advierte en que Pucara Río Negro y La Selva son fortalezas sobre colinas aisladas, mientras que el Remanso es un asentamiento habitacional anexo a caminos empedrados. Los sitios Bomboiza y Manabi Chico difieren de los anteriores porque están conformados por montículos. En Bomboiza los montículos son circulares y están dispuestos longitudinalmente uno tras otro (Ledergerber, 2006). Al margen de estos sitios, una serie de abrigos rocosos presenta material cerámico decorado en bandas rojas limitadas por líneas incisas, esta variedad de cerámica fue descrita inicialmente en Cerro Narrío por Collier y Murra (1943), mientras los restos cerámicos ubicados en las fortalezas tienen engobe rojo y son del tipo Tacalshapa-Cashaloma-Cañari (Ledergerber-Crespo, 2004). Las dataciones radiocarbónicas presentadas por Ledergerber-Crespo (2006: 152), muestran una concordancia entre las épocas de ocupación de los Abrigos Rocosos (1630 \pm 60 d.C.) y el sitio Remanso (1570 \pm 60 d.C.). Mientras la fecha de $1010 \pm 60$ d.C. de Pucara de Río Negro indica que su ocupación fue anterior. 
En la zona oriental de la provincia Zamora Chinchipe, las prospecciones arqueológicas realizadas por Valdez et al. (2005) en la región de Santa Ana-La Florida de la Amazonía Alta, dieron lugar al descubrimiento de un pequeño centro ceremonial «datado en el tercer milenio antes de Cristo», con estructuras funerarias y una cerámica con una pasta de color marrón rojiza decorada con corrugado, incisiones y puntuaciones, que podrían tener relación con la cerámica tardía de Loja (Valdez et al, 2005: 369-372).

En la Baja Amazonía del Sur (300 a 100 m snm), Ledergerber-Crespo (1995, 2006) menciona los sitios, Panientza, Cushapucu, Misión Santiago y Mayalico en la cuenca de los ríos Morona y Santiago, cada uno de ellos con evidencias de cerámica que parecen tener cierta influencia de la Fase Pastaza, aunque el sitio Mayalico está representado por una cerámica decorada en corrugado y falso corrugado. El sitio Cushapucu situado en la confluencia del Río Cushapucu con el Río Santiago tiene una datación de $1200 \pm 80$ d.C. (Ledergerber-Crespo, 2006: 152).

\section{Patrones de asentamiento}

Las investigaciones arqueológicas en la Amazonía del Ecuador muestran que las culturas prehispánicas tenían patrones de ocupación diferenciados según los medioambientes en los cuales se asentaron los pueblos prehispánicos. A lo largo de la Alta Amazonía las sociedades ocuparon, por una parte, los pocos y estrechos valles intermontanos de ceja de montaña conformando principalmente complejos estructurales asociados a montículos y por otra ocuparon colinas aisladas conformando una suerte de fortalezas con estructuras habitacionales al interior. Esta característica hace muy evidente las diferencias socioculturales entre los pueblos de ceja de la Alta Amazonía y la Baja Amazonía.

En la Alta Amazonía del Norte, la mayor parte de los sitios descritos como parte de la Fase Cosanga (Porras, 1975b) presentan restos de estructuras habitacionales dispuestas en amplias terrazas y ocasionalmente asociados a terrazas de cultivo (Salazar et al, 1999). Algunos sitios se encuentran integrados a rocas con petroglifos, considerados en la categoría de ceremoniales (Porras, 1975b; Salazar et al, 1999).

AlSur se pueden diferenciar dos tipos de patrón de ocupación. En el primero, los asentamientos se encuentran en valles relativamente amplios, y son complejos de montículos de formas variadas o plataformas dispuestas alrededor de una plaza central (Porras, 1987 y 1989; Salazar, 1999; Rostain, 1999b; Lerdergerber-Crespo, 2006). En el segundo tipo se aprecia un mayor contacto con las sociedades prehispánicas tardías de la sierra, por la ubicación de los asentamientos que ocasionalmente llegan a situarse por debajo de los $1000 \mathrm{msnm}$. Estos últimos sitios comprenden murallas y fortalezas con unidades habitacionales al interior (Ledergerber-Crespo, 2006; Valdez et al, 2005). 
Los patrones de asentamiento en la Baja Amazonía están con frecuencia asociados al curso de los principales ríos. Las investigaciones efectuadas por Netherly (1997) en el margen Sur del Napo, durante la construcción de uno de los importantes oleoductos del Ecuador, diferencian dos variedades de asentamientos, el primero denominado «de área ribereña» y el segundo «de loma». En el primer caso, los asentamientos se encuentran a lo largo de las riberas de los ríos con importantes caudales, aunque éstos no sean tributarios directos del Napo y en el segundo, los asentamientos se ubican en lomas aisladas al interior de la áreas interfluviales. La ubicación de una apreciable cantidad de sitios formando parte de estos dos patrones de asentamiento, dio lugar a un nuevo modelo para interpretar el uso de las zonas interfluviales por parte de los grupos amazónicos. Este modelo indica que no existiría una población concentrada en aldeas, sino por el contrario una gran población dispersa (Netherly, 1997: 34). Sin embargo, uno de los problemas de este modelo es la falta de correlación entre sitios arqueológicos mediante un estudio tipológico de la cerámica y fechas radiocarbónicas, para establecer si todos estos estuvieron ocupados en la misma época por una misma fase cultural.

El panorama al Norte del Río Napo se encuentra mucho más claro. Las investigaciones arqueológicas demuestran la existencia de otras fases culturales asociadas a los tributarios de la orilla izquierda del Río Aguarico. Si bien el patrón de asentamiento sigue en parte el modelo propuesto por Netherly (1997), existe una mayor diversidad en cuanto a la ocupación de micro-medioambientes. Por ejemplo, grupos específicos ocuparon pequeñas llanuras de inundación relacionadas con tributarios de los ríos Putumayo (frontera con Colombia) y Aguarico. Aquí algunos sitios fueron simplemente talleres líticos, mientras que otros constituían aldeas habitacionales y ceremoniales (Aguilera, 2002: 112). Los sitios que corresponden a este patrón se encuentran asociados a la sub-cuenca del Río San Miguel tributario del Putumayo, y las fechas radiocarbónicas indican que estuvieron ocupados entre el 1040 al 1290 d. C.

En ambas orillas del Aguarico, las evidencias arqueológicas se presentan en tres patrones de asentamiento: El primero, a lo largo de las terrazas altas anexas al río; el segundo, en la parte superior de las colinas alejadas del río principal y finalmente, el tercero, en pequeñas planicies asociadas a pequeñas lagunas. Es muy posible que la continua variabilidad de los patrones de ocupación en diferentes micro medioambientes, estuviera relacionada con los eventos climáticos que periódicamente y de manera alternada modificaban el paisaje. En la zona del Aguarico se determinó cronológicamente que las primeras ocupaciones son las que se ubican en las terrazas altas, luego que posteriormente en un segundo periodo se instalaron en las colinas y finalmente regresaron a ocupar áreas con terrazas bajas. Las fechas radiocarbónicas indican que las colinas fueron utilizadas como asentamientos entre los 1230 al 1330 d. C. (Arellano, 2002: 162). Por su parte, las 
terrazas altas fueron ocupadas entre el 1100 al $1200 \mathrm{~d}$. C. y las terrazas bajas entre el 1400 al 1500 d. C.

\section{Discusión y consideraciones finales}

La presente síntesis de las investigaciones arqueológicas llevadas a cabo en la Amazonía ecuatoriana, permite inferir en principio que las culturas asentadas en la Alta Amazonía y ceja de montaña, formaron una suerte de culturas marginales que se encontraron ocupando espacios geográficos de transición. En esta particular zona se puede observar que la geomorfología, tanto en el Norte como en el Sur, estuvo asociada a frecuentes cambios en su paisaje por efecto de la presencia de eventos medioambientales generados particularmente por la cadena de volcanes activos, los que paradójicamente proporcionaron material para una mejora en la calidad de los suelos. Es probable que esta característica diera lugar a la formación de asentamientos permanentes con acceso a los recursos naturales de la sierra y valles de ceja de montaña. En la Alta Amazonía, por el momento se ha determinado la presencia de dos importantes fases culturales: la Fase Cosanga en el Norte y la Fase Upano-Huapula al Sur, ambas fases con material cultural propio que no comparte ningún elemento diagnóstico tecnológico o decorativo.

En el caso de la Baja Amazonía, la concentración de investigaciones en la cuenca del Río Napo, demostró la existencia de una secuencia de fases culturales que por el momento parece iniciarse en el periodo de Desarrollos Regionales con la fase Yasuni. En esta cuenca, la definición de fases culturales, como se observa en el cuadro cronológico, está mucho más establecida que en la cuenca del Río Pastaza. En esta cuenca, la Fase Pastaza tiene un rango cronológico que va desde el Formativo al periodo de Integración, aspecto difícilmente comprensible por la situación geográfica del asentamiento. Esta discrepancia es observada por el material cerámico de Pumpuentza 1 que tiene diseños decorativos del material Pastaza. Sin embargo, las fechas C-14 calibradas para el sitio Huasaga, origen del material de la Fase Pastaza, confirman que pertenece al Formativo (Rostoker, 2003: 542).

Al margen de los tradicionales sitios que se encuentran en las orillas del Napo, las nuevas investigaciones demostraron que tanto los principales ríos tributarios del Napo como los ríos secundarios fueron ocupados por diversos grupos culturales. En consecuencia, el patrón de asentamiento en el periodo precolombino no solo estaba relacionado con las terrazas altas del Napo, sino también con las orillas de cada uno de los tributarios. De manera que el problema actual radica en la ubicación cronológica de estos grupos culturales prehispánicos.

Otro aspecto interesante se refiere a los complejos cerámicos recuperados. Los sitios en las terrazas altas usualmente tienen material cerámico que corresponde a un máximo de dos fases culturales sucesivas, mientras que los sitios en las colinas 
a una sola fase y en las terrazas bajas o planicies los complejos cerámicos son multicomponentes (Arellano, 2002; Carrera, 2002). En el caso particular de las terrazas altas se determinó que los sitios se fueron ampliando debido a un continúo movimiento de reocupación por familias que retornaban al sitio, pero que no construían la nueva vivienda sobre la anterior. Esta característica no se observa en los sitios que se encuentran en colinas donde el material cultural corresponde a una sola ocupación en la que también estaba incluido un espacio destinado a una agricultura en pequeña escala (Arellano, 2002).

Los sitios multi-componentes son más frecuentes en los límites con la Alta Amazonía, donde es posible ocasionalmente ubicar restos cerámicos derivados de las culturas serranas y de los primeros periodos de la ocupación española.

Las ultimas investigaciones en la Baja Amazonía han reportado la presencia de una cerámica decorada con corrugado y una variedad de diseños derivados del falso corrugado. Estos tipos que tienen un alto porcentaje de restos, se concentran principalmente al Norte del Río Napo y fueron interpretados como pertenecientes a la Fase Pastaza, tomando en cuenta algunos diseños decorativos descritos en la secuencia presentada por Porras (1975). Esta apreciación mencionada por arqueólogos que trabajan en la zona del Napo, no tiene la necesaria base empírica para considerar una difusión de estos particulares estilos decorativos desde el Sur hacia la cuenca del Napo. Otra perspectiva para la presencia de este tipo de cerámica en la Amazonía Norte del Ecuador, se genera a partir de los sitios con alto porcentaje de material cultural de estos tipos que se encuentran en la región comprendida entre los ríos Napo - Aguarico y con las evidencias obtenidas en la sub-cuenca del Río San Miguel, orilla derecha del Río Putumayo, se puede sugerir que la influencia para la adopción de esta variedad decorativa proviene del Norte (Aguilera 2002). Por otra parte, las dataciones radiocarbónicas indican que esta variedad de cerámica se ubica en una época tardía (1390 a 1440 d.C.) del periodo de Integración.

Este panorama de la arqueología amazónica de ninguna manera está completo, muchos informes de trabajos de campo son inaccesibles y no publicados. Aún así, se puede observar la compleja diversidad cultural que existió en la época prehispánica. En el medioambiente amazónico el desafío no solo se enfoca en el descubrimiento de nuevos sitios y una eventual profundización en su investigación, sino también en la integración de los resultados obtenidos por otras disciplinas que son parte de estudios medioambientales. La profundización en la investigación de los nuevos sitios registrados y determinación de fases culturales en los diferentes periodos de la cronología cultural, indudablemente llevará a conocer la secuencia en el entendido que los diferentes estilos decorativos en los restos de vasijas de cerámica mostraran las influencias y los canales interacción de las culturas del Bajo y Alto Amazonas con la sierra. 
La distribución geográfica de los asentamientos arqueológicos de la Alta Amazonía conforman los límites territoriales de las sociedades jerarquizadas, en las cuales el contacto con las sociedades de la Baja Amazonía se observa a través de los rasgos adoptados por la presencia de petroglifos y el empleo de urnas. Por otra parte, los datos arqueobotánicos que provienen de las investigaciones efectuadas en la cultura Upano-Huapula, pueden ser transferidas para considerar a estas sociedades como sedentarias y agrícolas. Las especies más frecuentes en las muestras de restos vegetales obtenidos en las excavaciones de Huapula, son el maíz (Zea mays) y la guaba (Inga sp.), que se complementan con algunas plantas medicinales y la inferencia del cultivo de algodón por la presencia de torteros (Rostain, 1999: 8184). Los contactos Andes y Alta Amazonía son más evidentes en la región Sur a través de la cerámica incisa en bandas rojas que aparecen en Pirincay (Bruhns, 1994, 2003: 146) y el valle del Río Upano (Porras, 1987; Rostoker, 1996; Rostain, 1999).

Por su parte, las evidencias arqueológicas en la Baja Amazonía sugieren sociedades móviles caracterizadas por la constante reocupación de sus asentamientos. Los grupos se movilizan a lo largo de los ríos, ya sea de los principales o de los tributarios. La concentración de evidencias arqueológicas en algunas áreas indica más que todo asentamientos de patrón disperso y no aldeas compactas. La asociación a una agricultura de subsistencia está sugerida por la frecuencia de rasgos de fogones con restos de semillas carbonizadas y vasijas coladores (Aguilera, 2002; Arellano, 2002; Carrera, 2002) ${ }^{2}$.

$2 \quad$ El autor desea expresar sus agradecimientos a la Escuela de Arqueología de la Universidad Mayor de San Marcos, en la persona del Señor Director Dr. Daniel Morales Chocano, al Centro Cultural José Pío Aza en la persona del Lic. Rafael Alonso, y a la Lic. Ana Mujica, Coordinadora del Simposio, por invitarme a participar en el Primer Simposio de Arqueología Amazónica del Perú.Así mismo, mis agradecimientos a la Dra. Betty J. Meggers, Dr. Dennis Stanford, Dr. Abelardo Sandoval y Lic. Paulina Lerdergerber del Smithsonian Institution por su constante amistad y apoyo.Este trabajo fue preparado, en parte, con los datos obtenidos en las investigaciones medioambientales en las que participé con Walsh Environmental Scientists and Engineers, Sección Ecuador. En este sentido, me permito agradecer a los señores gerentes Dr. Mark Turber y Ing. Peter Ayarza por todas las facilidades y amistad que me brindaron durante mi estadía en Ecuador. De la misma manera mis agradecimientos para el Dr. Ernesto Salazar y Myriam Ochoa por su apreciable amistad.Finalmente, me permito agradecer al Departamento de Antropologia del Museo de Historia Natural del Smithsonian Institution, que me dio la oportunidad de participar como Investigador Asociado. 


\section{Bibliografía}

\section{AGUILERA, $M$.}

2002 «Del San Miguel hacia el Norte». En: Cuyabeno Ancestral, pp. 49-122. Ediciones Simbioe. Quito.

\section{ARELLANO, A. J.}

2002 «Lago Agrio». En: Cuyabeno Ancestral, pp. 123-164. Ediciones Simbioe. Quito. 2007 Culturas Prehispánicas del Napo y Aguarico en la Amazonía Ecuatoriana. (En prensa).

\section{ATHENS, S.}

1984 «Pumpuentza 1, un sitio arqueológico cerca del Río Macuma en el oriente Ecuatoriano». En: Miscelánea Antropológica Ecuatoriana, No 4, pp. 129-140. Museo del Banco Central del Ecuador.

\section{BOLAÑOS, M.; MOREIRA, M.; MURILLO, R.; SANTAMARÍA, A.}

1999 Rescate Arqueológico en la Nueva Vía Interoceanica. Trabajo presentado a Constructora Norberto Odebrecht y Andrade Gutierrez. Instituto Nacional de Patrimonio Cultural del Ecuador. Quito.

\section{BRUHNS, K. O.}

1994 Ancient South America. Cambridge World Archaeology. Cambridge University Press.

2003 «Social and Cultural Development in the Ecuadorian Highlands and Eastern Lowlands during the Formative». In: J. Quilter. Archaeology of Formative Ecuador. pp. 125-174. Dumbarton Oaks Research Library and Collection. Washington D. C.

\section{CARRERA, J.}

2002 «Hacia el Suroeste de Nueva Loja». En: Cuyabeno Ancestral, pp. 165-203. Ediciones Simbioe. Quito.

\section{COLLIER, D.; MURRA, J.}

1943 «Survey and Excavations in Southern Ecuador». In: Anthropological Series, Vol. 35. Field Museum of Natural History. Chicago.

\section{DELGADO, F.}

1999 Prospección sistemática, rescate y monitoreo arqueológico del Proyecto de Desarrollo Campo Villano, Bloque 10. Informe al Instituto Nacional de Patrimonio Cultural del Ecuador. Quito. 


\section{ECHEVERRÍA, J.}

1992 «La cerámica prehispánica de la Región Oriental». En: Historia de la cerámica en el Ecuador. Pp. 29-32. Eds. Segundo Moreno y Jaime Peña. Fundación Paul Rivet \& Municipalidad de Cuenca. Cuenca.

1999 Informe Final del Proyecto de Investigación Arqueológica realizada en la Plataforma Pata 1, en el derecho de vía de la carretera y en la Plataforma Palo Azul del Bloque 18 de Cayman International Company, Región Amazónica Ecuatoriana. Instituto Nacional de Patrimonio Cultural del Ecuador. Quito.

EVANS, Cliford; MEGGERS, Betty J.

1968 Archaeological Investigations on the Rio Napo, Eastern Ecuador. Smithsonian Institution Press. Washington D. C.

HARNER, M. J.

1973 The Jivaro, People of the Sacred Waterfalls. Garden City. New York.

\section{LEDERGERBER-CRESPO, P.}

1992 Informe preliminar de la expedición Arqueológica a Morona Santiago de Agosto de 1991, para el Instituto Nacional de Patrimonio Cultural. Manuscrito en los Archivos del Smithsonian Institution. Washington DC.

1995 «Factores geográficos en la localización de sitios arqueológicos. El caso de Morona-Santiago, Ecuador. Un informe preliminar». En: Cultura y Medioambiente en el Area Andina Septentrional. Eds. M. Guinea, J. F. Bouchard y J. Marcos. pp. 343-375. Abya Yala. Quito.

2006 «Ecuador Amazónico-Andino: Apropiación de Paisajes y Relaciones Culturales». En: Pueblos y Paisajes Antiguos de la Selva Amazónica. Editores: Gaspar Morcote, Santiago Mora y Carlos Franky C. pp. 131-155. Universidad Nacional de Colombia. Taraxacum Washington DC.

MONCAYO, P.

1994 «Nuevas estructuras piramidales truncas en la margen izquierda del Río Upano, provincia Morona Santiago». En: Sarance, N ${ }^{\circ} 20$, pp. 147-154. Instituto Otavaleño de Antropología. Otavalo.

\section{NETHERLY, P.}

1997 «Loma y Ribera: Patrones de asentamiento prehistóricos en la Amazonía Ecuatoriana». En: Fronteras de Investigación, $\mathbf{N}^{\mathbf{0}}$ 1, pp. 33-54. Fundación Alejandro Von Humbolt. Quito.

\section{PORRAS GARCES, P.}

1975 (a) «El Formativo en el Valle Amazónico del Ecuador, Fase Pastaza». En: Revista de la Universidad Católica, Número Monográfico Arqueología III (10), pp. 74-134. Quito. 
1975 (b) Fase Cosanga. Ediciones de la Universidad Católica. Quito.

1978 Arqueología de la cueva de Los Tayos. Ediciones de la Universidad Católica del Ecuador. Quito.

1985 Arte Rupestre del Alto Valle de Misagualli, Ecuador. Imprenta Gráficas Señal. Quito.

1987 (a) Investigaciones arqueológicas a las faldas del Sangay, Provincia Morona Santiago, Fase Upano. Artes Gráficas Señal. Quito.

1987 (b) Nuestro Ayer. Manual de Arqueología Ecuatoriana. Centro de Investigaciones Arqueológicas-PUCE. Quito.

1989 «Investigations at the Sangay Mound Complex, Eastern Ecuador». In: National Geographic Research, No 5 (3), pp. 374-381. Washington DC.

\section{ROSTAIN, S.}

1999 (a) «Excavaciones en área en un montículo de Huapula, Amazonía Ecuatoriana». En: Memorias del Primer Congreso Ecuatoriano de Antropología, Vol. III, pp. 227-256. Museo Jacinto Jijón y Caamaño. PUCE.

1999 (b) «Secuencia arqueológica en montículos del valle de Upano en la Amazonía Ecuatoriana». En: Boletín del Instituto Francés de Estudios Andinos, $\mathrm{N}^{\circ} 28$ (1), pp. 53-89. Lima.

\section{ROSTOKER, A.}

1996 An Archaeological Assemblage from Eastern Ecuador. Traganza Anthropology Museum Papers 18. San Francisco State University.

2003 «Formative Period Chronology for Eastern Ecuador». En: Archaeology of Formative Ecuador. General Editor: J. Quilter, pp.539-545. Dumbarton Oaks Research Library and Collection. Washington D. C.

SALAZAR, E.; ARELLANO, J.; OCHOA, M.; MANOSALVAS, O.

1999 Informe del Reconocimiento Arqueológico de la Línea del Oleoducto Ecuatoriano y Zonas Adyacentes. Instituto Nacional de Patrimonio Cultural. Quito. Ecuador.

SALAZAR, E.

1999 «De vuelta al Sangay: Investigaciones Arqueológicas en el Alto Upano». En: Memorias del Primer Congreso Ecuatoriano de Antropología, Vol. III, pp. 183-226. Museo Jacinto Jijón y Caamaño. PUCE.

VALDEZ, F.; GUFFROY, J.; DE SAULIEU, G.; HURTADO, J.; YEPES, A.

2005 «Découverte d' un site cérémoniel formativ sur le versant oriental des Andes». En: Paleovol, No 4 . Centre IRD-Quito. Elsevier, SAS. 


\section{Amazonía Peruana}

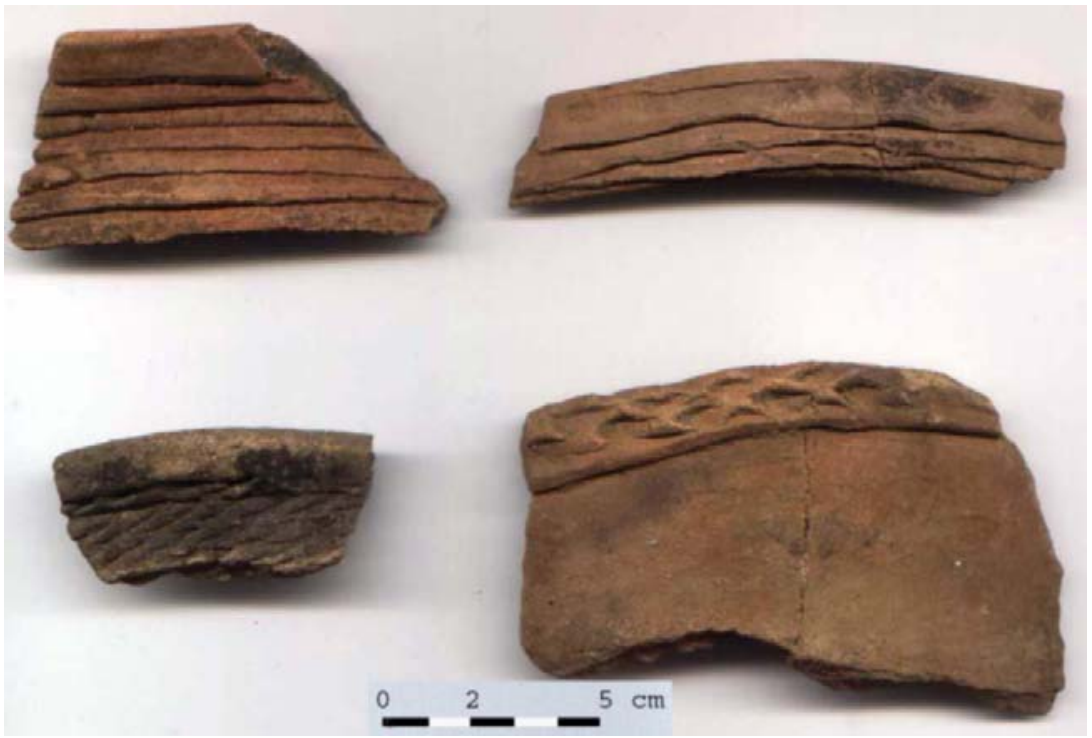

Figura 3.

Fragmentos de cerámica decorada del sitio UNE, Lago Agrio.

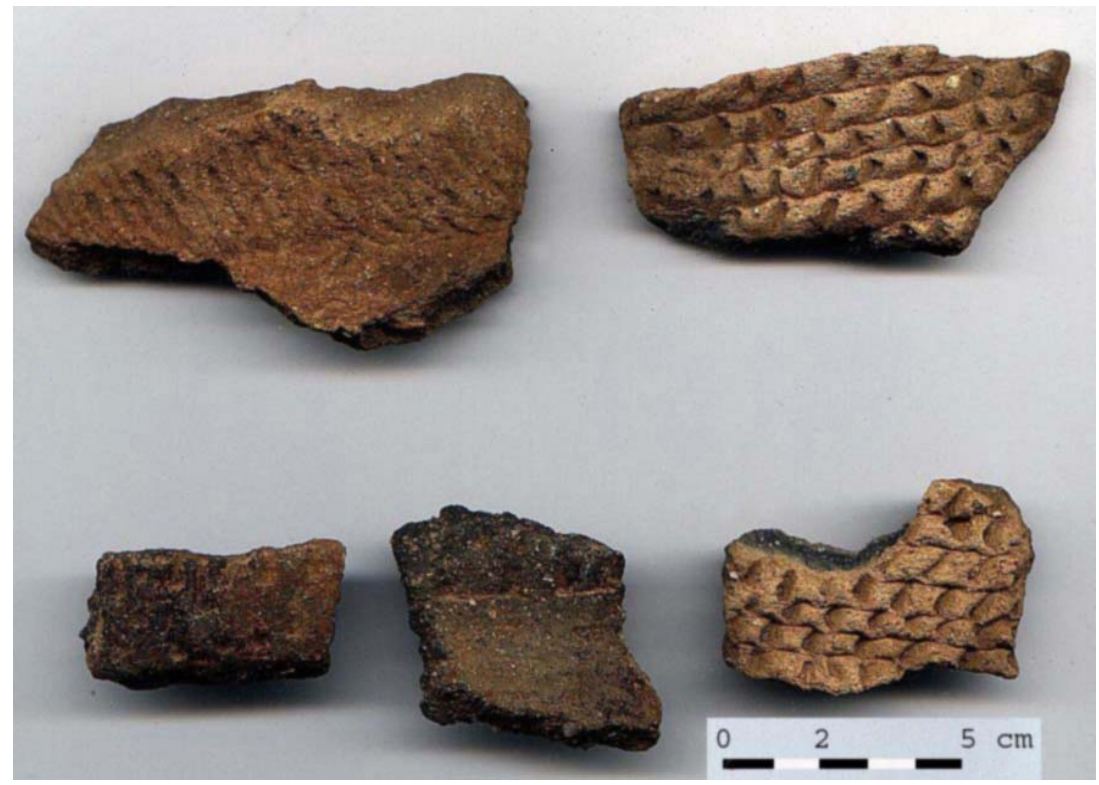

Figura 4.

Fragmentos de cerámica decorada con puntuaciones.

Sitio Tank Farm, Lago Agrio. 


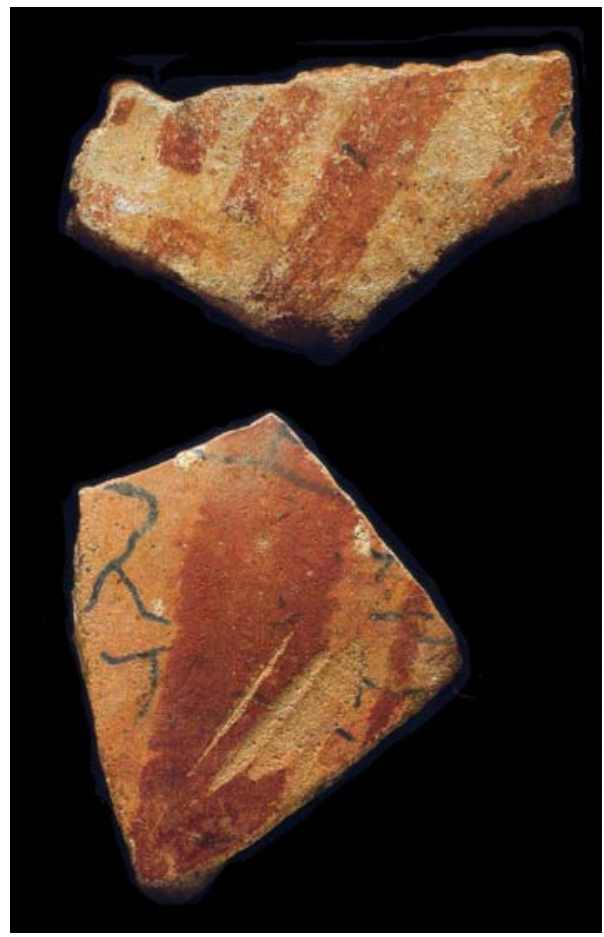

Figura 5.

Fragmentos decorados con pintura roja sobre crema. Sitio UNE, Lago Agrio.

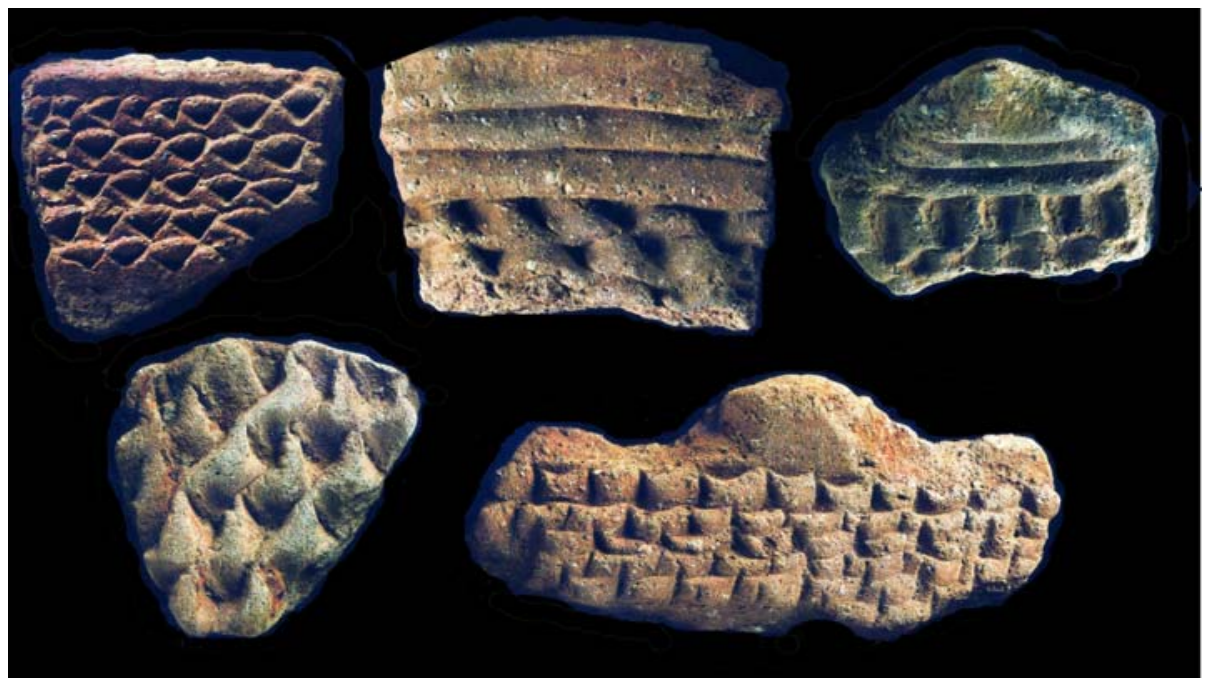

Figura 6.

Fragmentos con decoración corrugada compleja. Sitio UNE, Lago Agrio. 


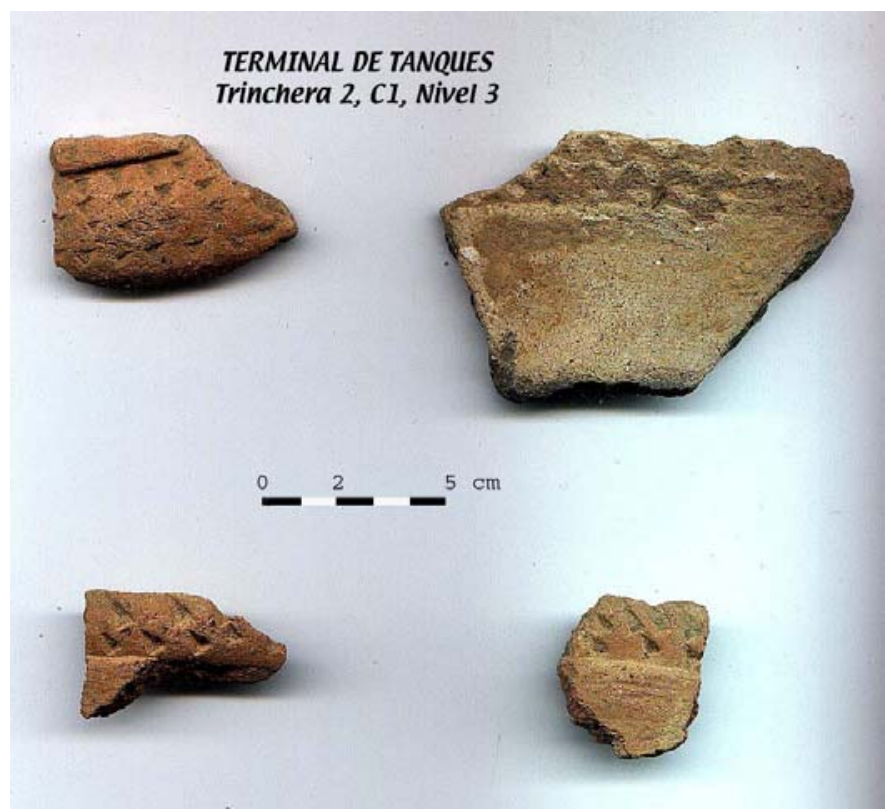

Figura 7.

Fragmentos decorados con puntuación. Sitio Terminal de Tanques, Lago Agrio.

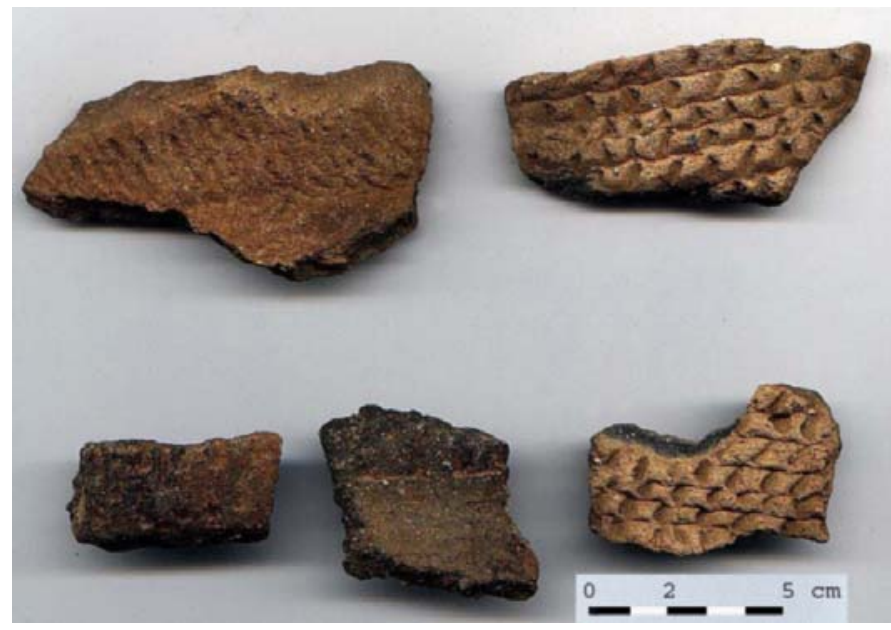

Figura8.

Fragmentos con decoración corrugada.

Sitio Mayalico, Gualaquiza. (Gentileza Dra. P. Lerdergerber) 